\title{
Dual-harmonic auto voltage control for the rapid cycling synchrotron of the Japan Proton Accelerator Research Complex
}

\author{
Fumihiko Tamura* and Alexander Schnase \\ J-PARC Center, Japan Atomic Energy Agency, 2-4 Shirakata-Shirane, Tokai, Naka, Ibaraki, Japan 319-1195
}

Masahito Yoshii

J-PARC Center, KEK, 1-1 Oho, Tsukuba, Ibaraki, Japan 305-0801

(Received 13 March 2008; published 17 July 2008)

\begin{abstract}
The dual-harmonic operation, in which the accelerating cavities are driven by the superposition of the fundamental and the second harmonic rf voltage, is useful for acceleration of the ultrahigh intensity proton beam in the rapid cycling synchrotron (RCS) of Japan Proton Accelerator Research Complex (J-PARC). However, the precise and fast voltage control of the harmonics is necessary to realize the dual-harmonic acceleration. We developed the dual-harmonic auto voltage control system for the J-PARC RCS. We describe details of the design and the implementation. Various tests of the system are performed with the RCS rf system. Also, a preliminary beam test has been done. We report the test results.
\end{abstract}

DOI: 10.1103/PhysRevSTAB.11.072001

PACS numbers: $29.20 .-\mathrm{c}$

\section{INTRODUCTION}

Japan Proton Accelerator Research Complex (J-PARC) $[1,2]$ is a project of a high-intensity proton accelerator complex, which consists of $181 \mathrm{MeV}$ linac (to be $400 \mathrm{MeV}$ in the energy-recovery plan), $3 \mathrm{GeV}$ rapid cycling synchrotron (RCS), and $50 \mathrm{GeV}$ synchrotron (MR). Since the beam power of the RCS is to be very high, $1 \mathrm{MW}$ with the full design energy of the linac, it is important to accelerate the beams with minimal losses, which cause activation of the accelerator components. The activation must be kept low so that hands-on maintenance is possible.

The parameters of the rf system of the RCS are listed in Table I. In the RCS the proton beam is accelerated from $181 \mathrm{MeV}$ to $3 \mathrm{GeV}$ in $20 \mathrm{~ms}$. The repetition rate is $25 \mathrm{~Hz}$. The maximum rf voltage is $450 \mathrm{kV}$ per turn. To generate the high accelerating voltage in the limited space of the ring, we employ magnetic-alloy (MA) loaded cavities. The MA-loaded cavity can achieve a very high accelerating field at the level of $25 \mathrm{kV} / \mathrm{m}$, which is more than twice of the field achieved by ferrite-loaded cavities $(10 \mathrm{kV} / \mathrm{m})$.

The harmonic number is chosen as 2 and the accelerating frequency sweeps from 0.94 to $1.67 \mathrm{MHz}$. The cavity is adjusted to have the Q-value of 2. It is a wideband cavity and does not require a tuning loop to cover the accelerating frequency sweeps. Also, the wideband frequency response allows dual-harmonic operation. In the dual-harmonic operation, a single cavity is driven by a superposition of the rf signals, the fundamental accelerating of $(h=2)$, and the second harmonic rf $(h=4)$. The second harmonic $(h=$ 4 ), which modifies the rf bucket shape, is used for the longitudinal bunch shape control.

The space charge effects must be considered for the acceleration of the high beam current and the effects are

*fumihiko.tamura@j-parc.jp especially severe in the injection period. By increasing the bunching factor, which is defined by the ratio between average and peak current, we can alleviate the space charge effects. We combine the momentum-offset injection [3] and the dual-harmonic operation for increasing the bunching factor. In Fig. 1 a typical bunch shape simulated using these schemes is illustrated. In the simulation, the second harmonic voltage ratio to the fundamental is $80 \%$ at maximum. The simulated bunch is very flat and the bunching factor is large, 0.4 .

As described above, the dual-harmonic operation is essential for the stable acceleration of ultrahigh intensity beams. Typical voltage programs for the RCS are shown in Fig. 2. The voltage ratio of the accelerating $\mathrm{rf}(h=2)$ and the second harmonic varies in the RCS cycle. Also, the impedance of the MA-loaded cavity with no tuning changes according to the frequency sweeps, as shown in Fig. 3. For the fundamental harmonic $(h=2)$, the cavity impedance varies from $126 \Omega$ to $288 \Omega$ in $20 \mathrm{~ms}$. Therefore the dual-harmonic auto voltage control (AVC),

TABLE I. Parameters of the J-PARC RCS rf.

\begin{tabular}{lc}
\hline \hline Circumference & $348.333 \mathrm{~m}$ \\
Energy & $0.181-3 \mathrm{GeV}$ \\
Accelerating frequency & $0.94-1.67 \mathrm{MHz}$ \\
Harmonic number & 2 \\
Maximum rf voltage & $450 \mathrm{kV}$ \\
Repetition period & $40 \mathrm{~ms}$ \\
Duty (power) & $30 \%$ \\
Number of cavities & $11^{\mathrm{a}}$ \\
Q-value & 2 \\
Number of gaps & 3 per cavity \\
Average power & $120 \mathrm{~kW} /$ cavity \\
\hline \hline
\end{tabular}

${ }^{\mathrm{a}}$ This is the design number. The day-1 operation started with 10 cavities. 

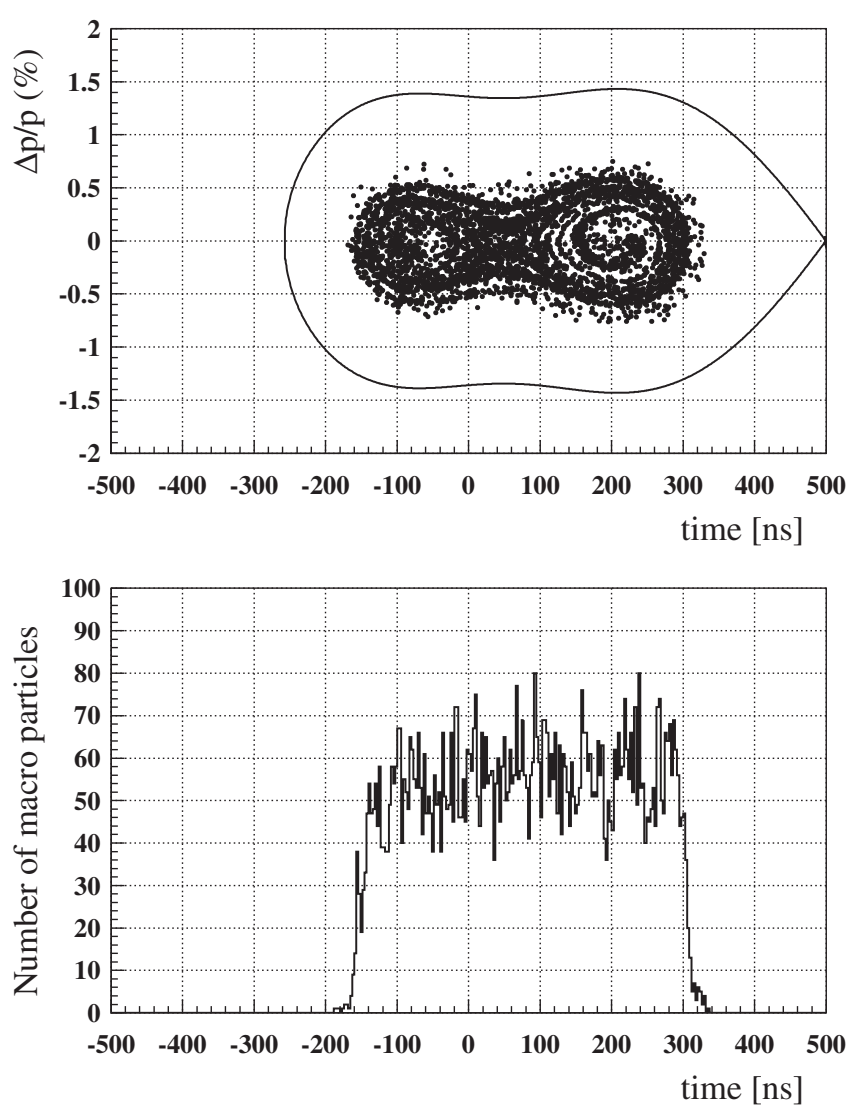

FIG. 1. A typical bunch shape after injection applying the second harmonic rf. The ratio of the second harmonic to the fundamental is $80 \%$.

which controls the voltage of $(h=2)$ and $(h=4)$ independently, is necessary to follow the RCS voltage programs.

We designed and built the dual-harmonic AVC as a part of the low level rf (LLRF) control system of the J-PARC RCS. We also performed tests with the high power rf

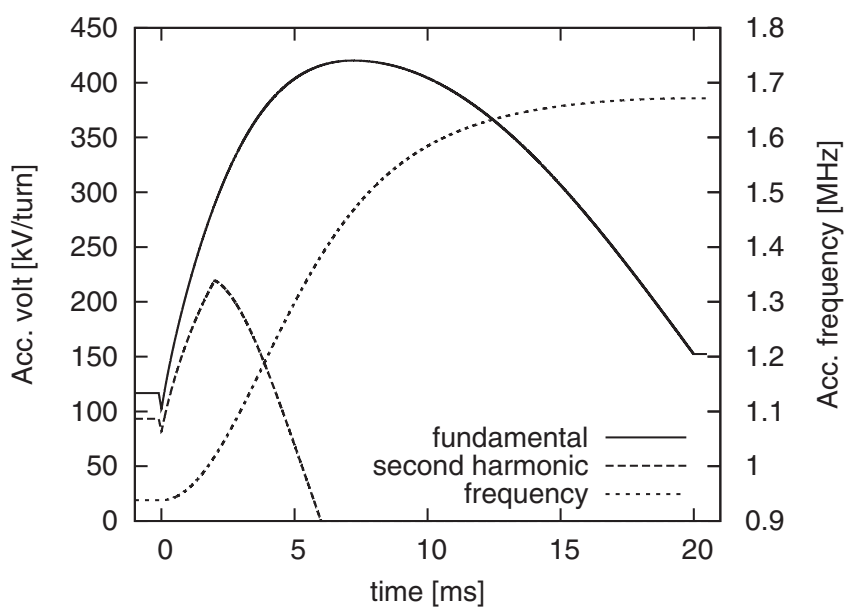

FIG. 2. Typical rf frequency and voltage programs of the RCS used in the longitudinal tracking simulation.

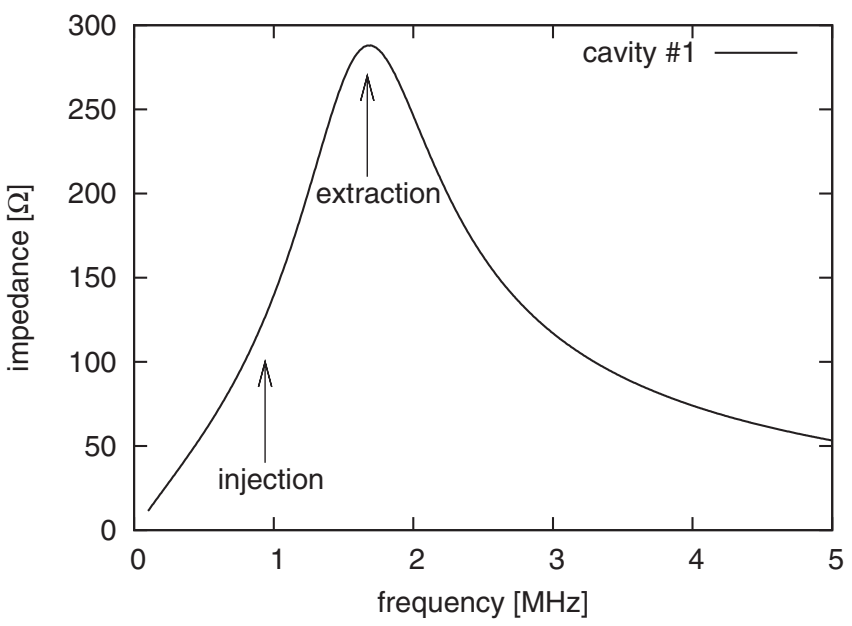

FIG. 3. Typical rf cavity impedance (absolute value, cavity \#1).

system, which includes the MA-loaded cavity and the 1 MW final-stage amplifier.

In Sec. II we present details of the design and implementation of the dual-harmonic AVC. We have performed tests with the high power rf system of the RCS. The results are reported in Sec. III. We make some concluding remarks and points to future work in Sec. IV.

\section{DESIGN AND IMPLEMENTATION OF THE DUAL-HARMONIC AVC}

\section{A. LLRF system overview}

A simplified block diagram of the main part of the LLRF system [4] is shown in Fig. 4.

The LLRF system is a full-digital system. The system clock is chosen as $36 \mathrm{MHz}$. The system clock is generated by locking to the J-PARC master clock at the frequency of $12 \mathrm{MHz}$, which is distributed from the central control room of the J-PARC. By locking to the master clock, the LLRF is synchronized with the J-PARC timing system and the other accelerator components.

The key feature of the LLRF system is the multiharmonic direct digital synthesis (DDS) implemented in the "standard-phase generator (SPG)" module. The multiharmonic rf signals are generated for driving the cavities and for the reference of the rf signal detection $(I / Q$ modulation/demodulation). A schematic of the DDS is shown in Fig. 5. A phase increment is fetched from the frequency pattern memory at each pattern-clock cycle, and the phase accumulator generates a sawtooth phase signal at the beam revolution frequency. By basic arithmetic operation, higher harmonic phase signals are generated. The DDS can generate the multiharmonic signals without phase lock loop and the revolution frequency signal and the higher harmonic signals are easily synchronized. The rf signal is generated by converting the phase signal into a sine wave by coordinate transformation. For the dual-harmonic AVC, 


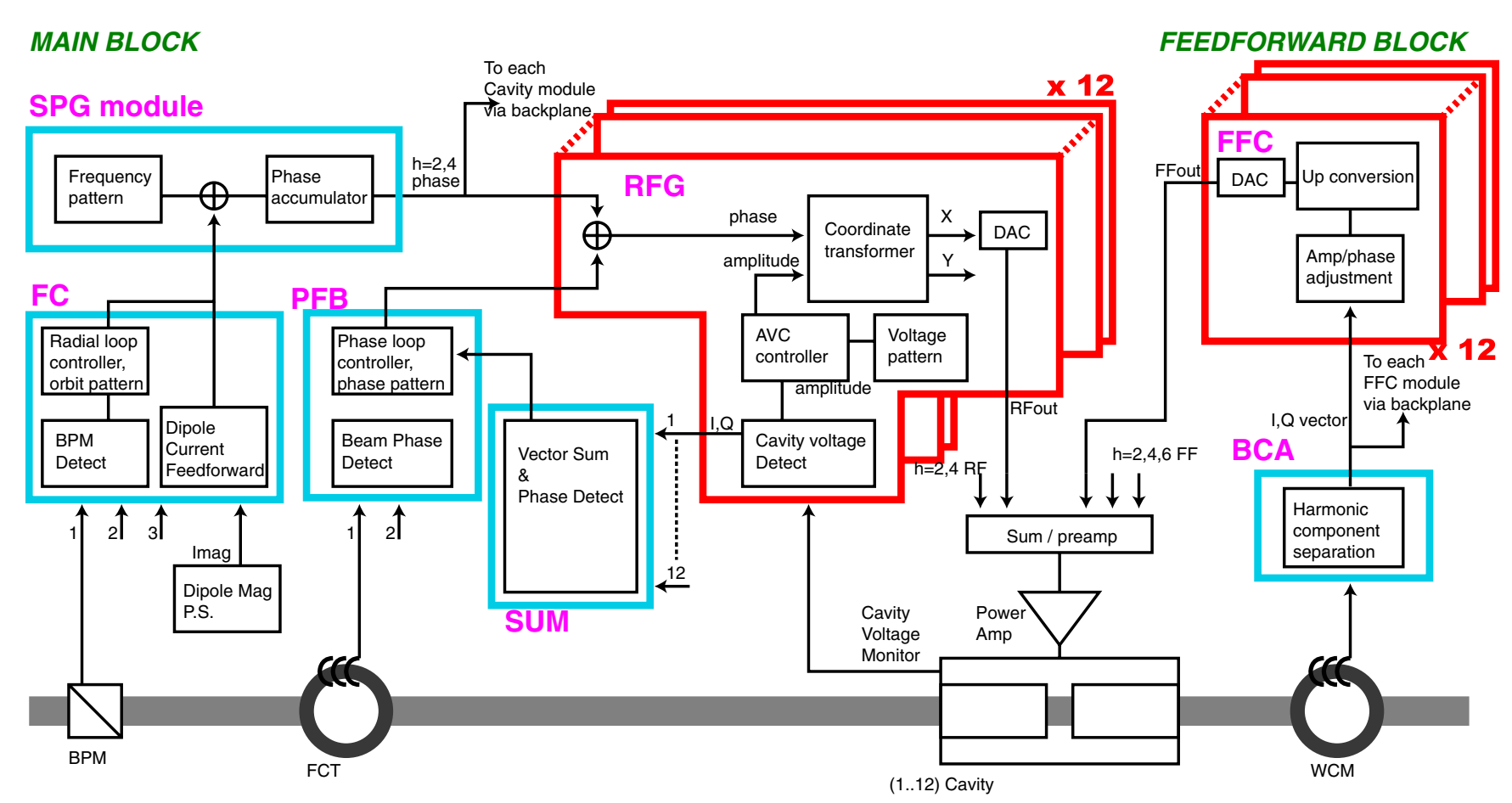

FIG. 4. (Color) Simplified block diagram of the LLRF system of the RCS. Red: for each system; blue: one for whole system.

the $(h=2)$ and $(h=4)$ phase signals are used. The phase reference signals are distributed to the LLRF modules.

We employ common beam feedback loops. The radial loop controls the rf frequency referring to the beam orbit. Three beam position monitors (BPM) are used and the signals from the BPMs are averaged with proper weights. By summing up several BPM signals, we can cancel the effects of the closed orbit distortion (COD) at the BPMs. The phase loop is for damping the dipole oscillations of the bunch. The phase loop compares the beam phase picked up by fast current transformers (FCT) and the vector sum of the rf voltage of the cavities. In Fig. 4 the "phase feedback (PFB)" and "frequency control (FC)" module are for the phase feedback and the radial loop, respectively. The "beam current analysis (BCA)" and "feedforward cavity driver (FFC)" modules are for the beam-loading compensation by the beam feedforward method [5].

The dual-harmonic AVC is implemented in the "rf generator (RFG)" module. Each cavity system has a corresponding RFG module. Details of the dual-harmonic AVC are discussed in the following subsections.

\section{B. Design of dual-harmonic AVC}

The block diagram of the dual-harmonic AVC is shown in Fig. 6. As described in the previous subsection, the dualharmonic AVC is also designed as a full-digital system.

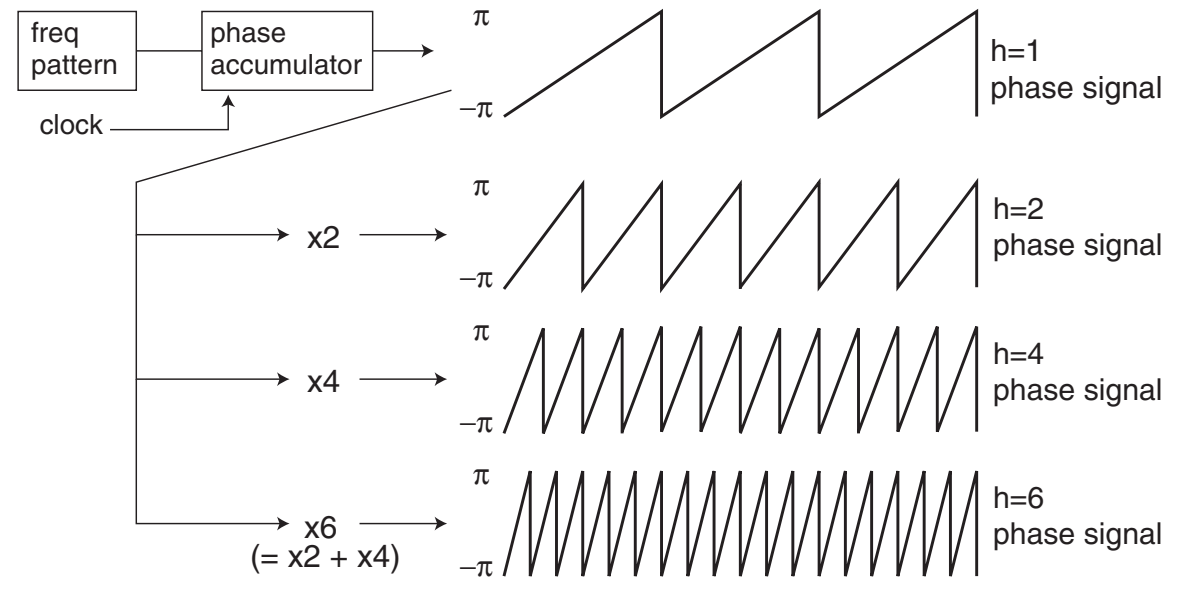

FIG. 5. Direct digital synthesis (DDS). 


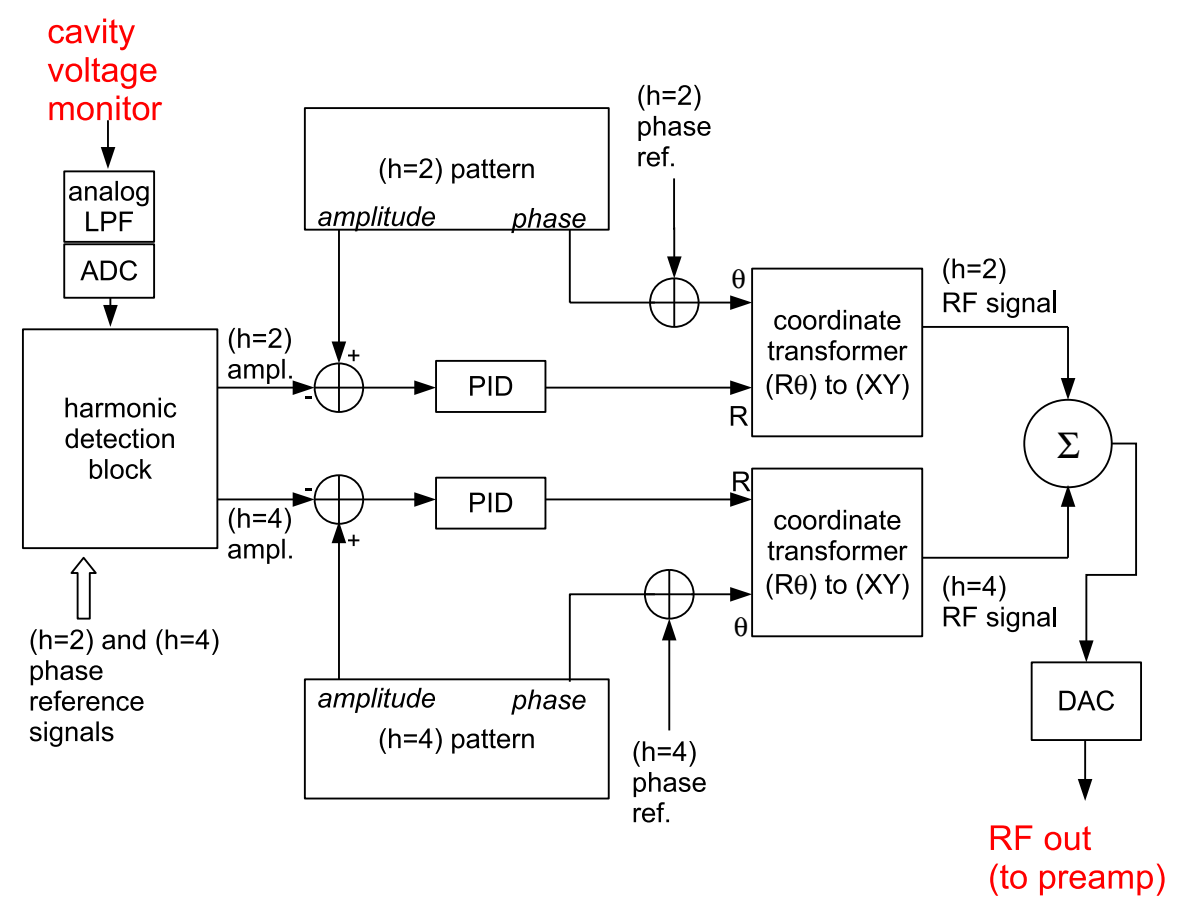

FIG. 6. (Color) Block diagram of the dual-harmonic AVC.

The input rf signal from the cavity voltage monitor is converted into a digital signal by the analog-to-digital converter (ADC). The ADC sample frequency is $36 \mathrm{MHz}$. The digitized signal is then led into the harmonic-detection block. In this block, the amplitudes of the fundamental $(h=2)$ and the second harmonic $(h=$ 4) are detected by using the $(h=2)$ and $(h=4)$ phase reference signals, which are generated in the SPG module and distributed to the RFG modules via the backplane.

The detail schematic of the harmonic-detection block is illustrated in Fig. 7. The block consists of two minor $(h=$ $2)$ and $(h=4)$ detection blocks, which have a similar structure. The $I / Q$ demodulation technique is used for the harmonic detection. The cavity voltage signal is multiplied by cosine and sine signals of unity amplitude. The signals are generated in a look-up table (LUT) by using the $(h=2)$ or $(h=4)$ phase reference signal. The multiplied signals are led into low-pass filters (LPF).

The cavity voltage signal contains not only the driven harmonics, $(h=2)$ and $(h=4)$, but also the beam induced harmonics. In the case of the one-bunch operation, the beam revolution frequency $(h=1)$, and its odd harmonics $(h=3)$ and $(h=5)$ are induced in the cavity. The LPF must reject the nearest of the selected harmonics. For the detection of $(h=2)$ the nearest harmonics are $(h=1)$, and $(h=3)$, and for $(h=4)$ the nearest ones are $(h=3)$ and $(h=5)$. As shown in Table I, the frequency separation of the harmonics in the J-PARC RCS is smallest at the injection, $0.47 \mathrm{MHz}$. Therefore we designed the cutoff frequency of the LPF as $0.2 \mathrm{MHz}$, which is narrow enough to reject $0.47 \mathrm{MHz}$ completely.
After the LPF, the $I / Q$ signals contain only the information of the selected harmonics. The $I / Q$ signals $I_{(2,4)}$ and $Q_{(2,4)}$ of the $(h=2,4)$ are represented as

$$
I_{(2,4)}=A_{(2,4)} \sin \left(\phi_{(2,4)}\right)
$$

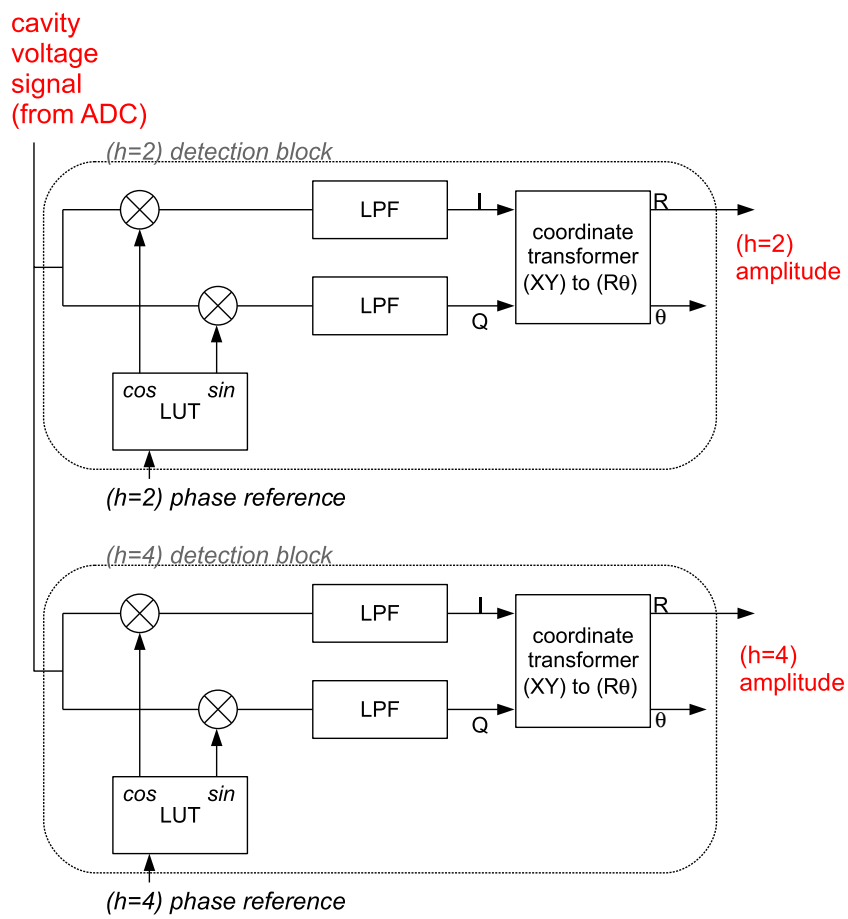

FIG. 7. (Color) Details of the harmonic-detection block. 


$$
Q_{(2,4)}=A_{(2,4)} \cos \left(\phi_{(2,4)}\right)
$$

where $A_{(2,4)}$ and $\phi_{(2,4)}$ are the amplitude and the phase of the harmonics $(h=2,4)$, respectively.

The $I / Q$ signals are then input to the coordinate transformer, which converts $(X, Y)$ to $(R, \theta)$. The $R$ output is the amplitude of the selected $(h=2)$ or $(h=4)$ component.

The amplitudes of $(h=2)$ and $(h=4)$ are compared with the amplitude patterns. The differences are led into proportional-integral-derivative (PID) controllers. By using the output of the PID controller and the phase signal, the coordinate transformer, which converts $(R, \theta)$ to $(X, Y)$ in this case, generates the rf signal of the selected harmonic. The phase signal is the sum of the phase reference signal and the phase pattern, which corresponds to the phase differences of the cavities due to the position in the RCS ring.

Finally, the $(h=2)$ rf signal and the $(h=4)$ signal are summed to generate the dual-harmonic rf signal. The digital-to-analog converter (DAC) output signal is sent to the amplifier to drive the cavity.

\section{Implementation details}

We implement the LLRF system for the J-PARC RCS as a full-digital system with high-performance FPGA (XILINX Virtex-II). The strong computing power of FPGA, especially of its multiplier logic cells, is necessary to implement the digital low-pass filter, which is a key element of the harmonic-detection blocks.

As described in the previous section, the minimum separation of the harmonic signal is $0.47 \mathrm{MHz}$ and the designed bandwidth of the low-pass filter in the harmonicdetection block is $200 \mathrm{kHz}$. As illustrated in Fig. 8, the lowpass filter is composed of a series of a cascade of integrator and comb (CIC) filter and a 63-tap interpolated finite impulse response (IFIR) filter [6].

The CIC decimation factor is 4 , so that the IFIR is running at $9 \mathrm{MHz}$. The IFIR is designed to have a linearphase response.

The frequency response of the low-pass filter is illustrated in Figs. 9 and 10. In the figure, the individual response of the CIC and FIR filters is also shown. The rejection at the $0.47 \mathrm{MHz}$ is more than $-100 \mathrm{~dB}$, and the passband ripple is small.

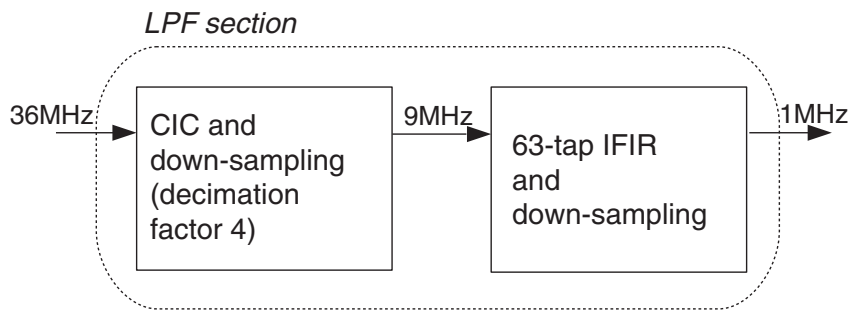

FIG. 8. LPF composed of a series of CIC and FIR.
The LPF delay is the major part of the overall loop latency. The CIC has approximately $1 \mu$ s delay and the IFIR has $14 \mu \mathrm{s}$. By summing up the group delay of the PID

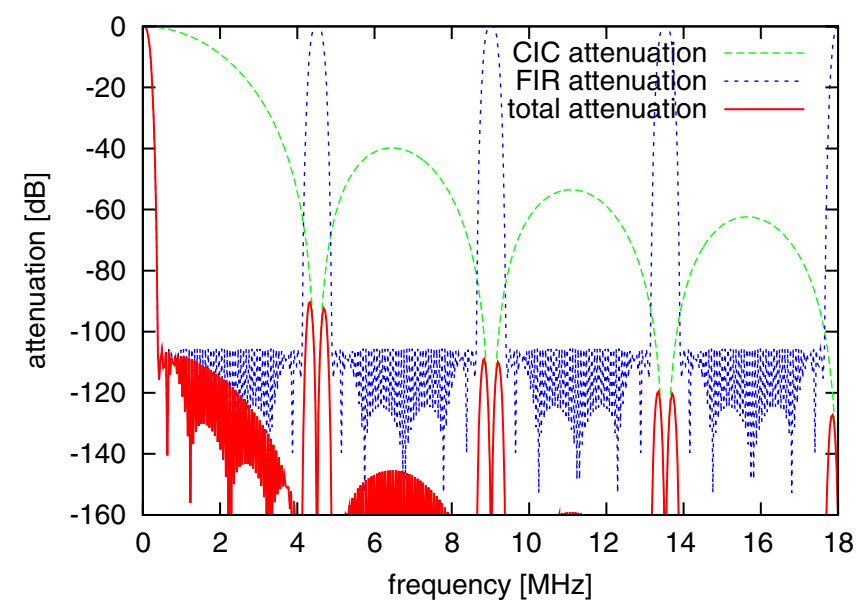

FIG. 9. (Color) Frequency response of the LPF in the harmonicdetection block.

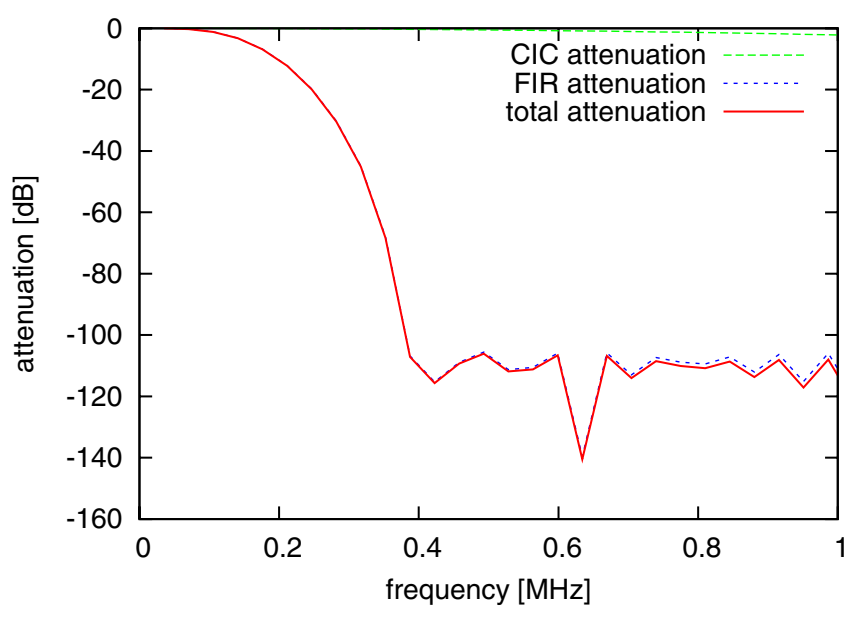

FIG. 10. Frequency response of the LPF in the harmonicdetection block (magnified). The attenuation at $470 \mathrm{kHz}$ is more than $-100 \mathrm{~dB}$.

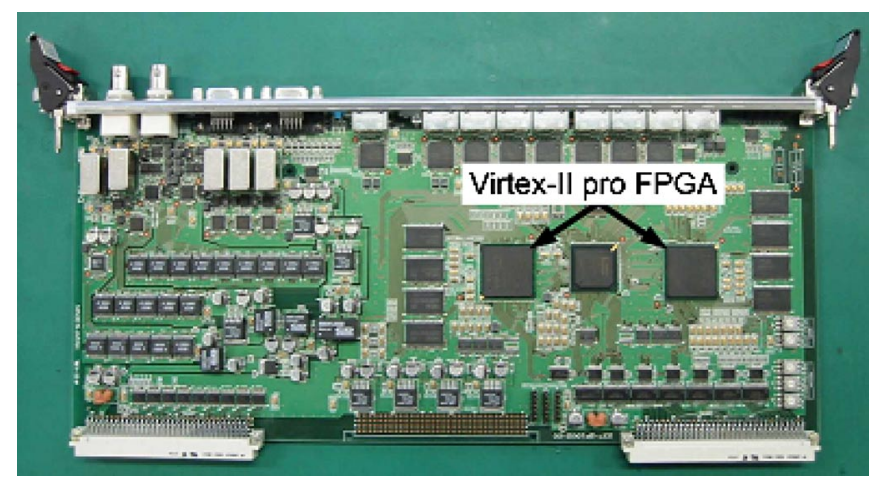

FIG. 11. (Color) RFG board. 
section, the up-sampling part, and the analog path (the cables, the transistor and tube amplifiers, and the cavity), the overall loop latency is approximately $20 \mu \mathrm{s}$.

The system bandwidth is basically defined by the LPF, which has its $-3 \mathrm{~dB}$ point at $140 \mathrm{kHz}$. The other components such as ADC and DAC, amplifiers, and cavity have much wider bandwidth and can be neglected for the bandwidth estimation.

The photograph of the RFG board is shown in Fig. 11. On the board we have two Virtex-II FPGAs in which the $(h=2)$ and $(h=4)$ AVC are implemented. We employ the 9U-height VME (versa module Europe) to mount many $\mathrm{I} / \mathrm{O}$ ports on the front panel.

\section{TEST RESULTS}

\section{A. Configuration}

Using the rf systems of the J-PARC RCS [7], we have tested the dual-harmonic AVC.

The configuration of the RCS rf system is illustrated in Fig. 12. The rf signal from the LLRF system is led to the transistor driver amplifier, which is located in the power supply room on the ground floor. The output signal of the driver amplifier is then led to the final-stage tube amplifier in the RCS tunnel. The maximum power dissipation of the anodes of the tubes in the final-stage amplifier is in the order of $1 \mathrm{MW}$. As described in Sec. I, we employ the accelerating cavity loaded with MA cores. By using MA cores, we can achieve high accelerating voltages up to $45 \mathrm{kV}$ per cavity.

The cavity voltages are monitored by capacitive dividers, which have a voltage ratio of 2000:1. Each of the upstream and downstream sides of the center accelerating gap has its own voltage monitor. The signals are then combined by a $0-\pi$ combiner in the rf control room on the ground floor. The combined signal is proportional to the gap voltage seen by the proton beam. We employ rf transformers to reject the low-frequency common mode noise. By attenuators the signal level is fitted to the RFG board specification, $2 \mathrm{~V}$ peak-peak.
The rf system gain widely varies due mainly to the frequency response of the cavity. As described in Sec. I, the cavity impedances for the fundamental accelerating harmonic in the injection and extraction periods are $126 \Omega$ and $288 \Omega$, respectively. Also, the gain of the tube amplifier has a small amplitude dependence. As described in Sec. I, the wide amplitude modulation is necessary to accelerate the beam. Therefore, the system gain changes in the accelerating cycle. Typical numbers are as follows: (i) To generate an accelerating gap voltage of $21.2 \mathrm{kVpp}$ at $1.7 \mathrm{MHz}$, the RFG output amplitude of " $17989 "$ ( $0.5 \mathrm{~V}$ corresponds to 32768$)$ is necessary. This case the detected amplitude of the cavity signal is "16374". Thus, the rf system gain is 0.910 . (ii) To generate a gap voltage of $8.5 \mathrm{kVpp}$ at $0.938 \mathrm{MHz}$, the RFG output amplitude of " 14352 " is necessary and the detected amplitude is " 6540 ". Therefore, the system gain is 0.456 . We explored the PID gain and finally reached the stable condition of that the proportional gain is 0.36 , the integral gain is 0.106 and the differential is 0 .

We took the waveforms of the voltage monitor signals by an oscilloscope, and recorded the amplitude monitor output signals of the RFG for both $(h=2)$ and $(h=4)$ components, and the amplitude signals are compared with the programs.

The phase of the $(h=4)$ signal is locked to the phase of the fundamental $(h=2)$ phase of the cavity voltage, by enabling the phase feedback loop of the second harmonic rf voltage.

\section{B. Test with fixed ratio of the harmonics}

We set the gap voltage of both of the $(h=2)$ and $(h=$ 4) harmonics as $9.8 \mathrm{kVpp}$. In this case, the ratio of the harmonics is 1:1. The waveforms of the single-harmonic rf and the superposed rf signal are taken by an oscilloscope at various frequencies. In this test, the phase offset of the second harmonic is set to 0 -degree to the fundamental $\mathrm{rf}$ voltage by the phase feedback loop.

The waveforms at the frequency of 0.9 and $1.5 \mathrm{MHz}$ are shown in Figs. 13 and 14, respectively.

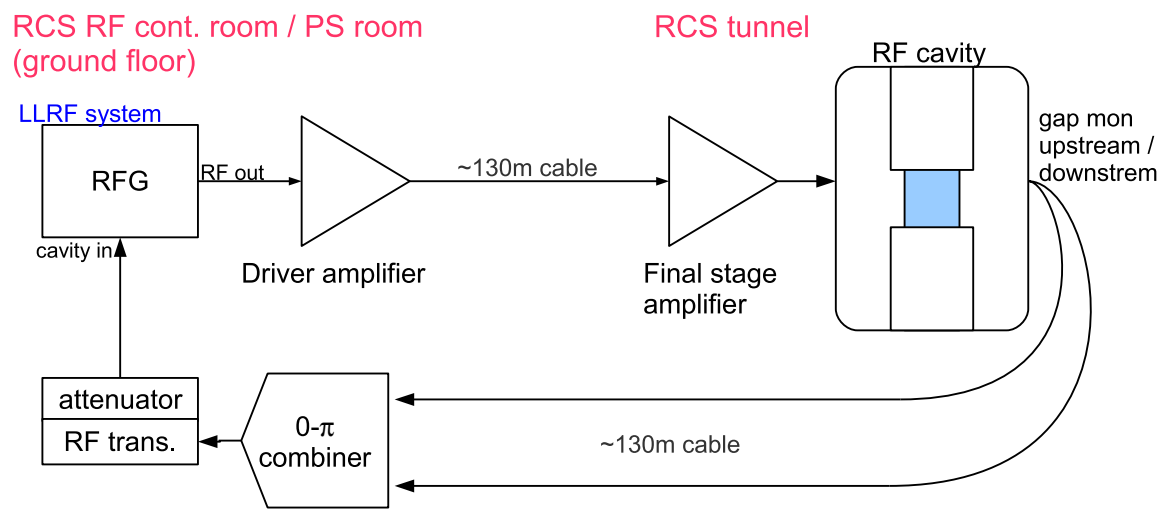

FIG. 12. Configuration of the RCS rf system. 


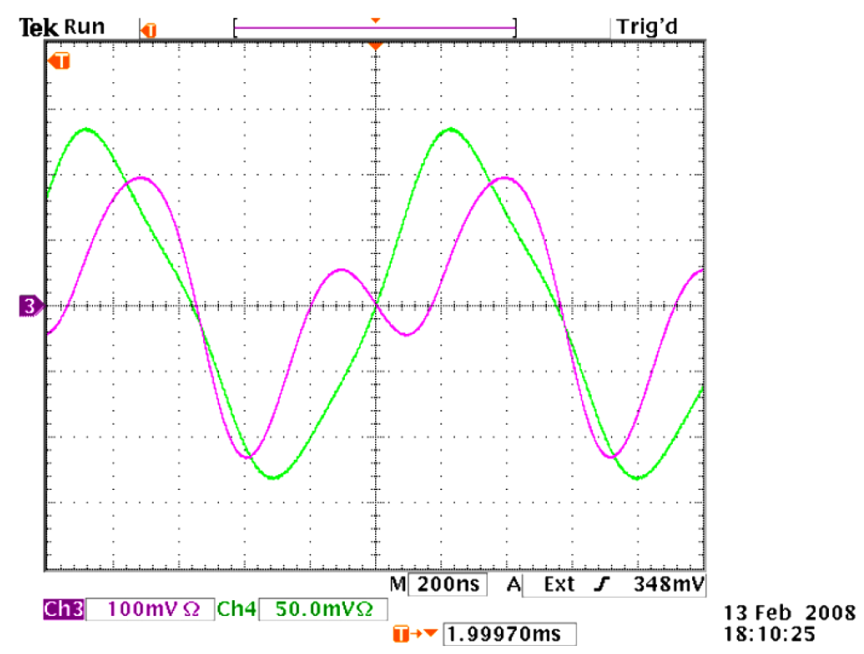

FIG. 13. (Color) Fundamental rf frequency is $0.9 \mathrm{MHz}$. Superposed rf signal (channel 3) and single harmonic waveform (channel 4).

In case of $0.9 \mathrm{MHz}$, the signal contains the third harmonic component about $10 \%$ in amplitude to the fundamental, due to the natural distortion of the tetrode amplifier in class $\mathrm{AB}$. In case of $1.5 \mathrm{MHz}$, the distortion is much smaller because we are more near to the cavity resonance peak. In both cases, the ratio between the fundamental and the second harmonic is very close to $1: 1$. This shows that the dual-harmonic AVC is working properly.

\section{Dual-harmonic accelerating pattern}

We also performed the test with the dual-harmonic acceleration pattern. The pattern used in the test is shown in Fig. 15, which is different from the pattern shown in Fig. 2. The differences are the peak voltage and the ratio of $(h=2)$ and $(h=4)$, modified because of the practical

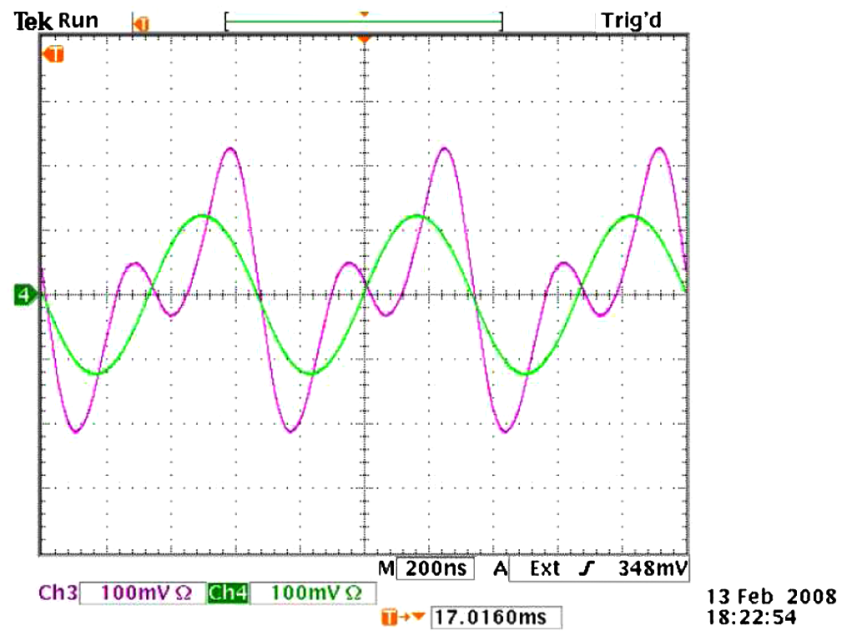

FIG. 14. (Color) Fundamental rf frequency is $1.5 \mathrm{MHz}$. Superposed rf signal (channel 3) and single harmonic waveform (channel 4).

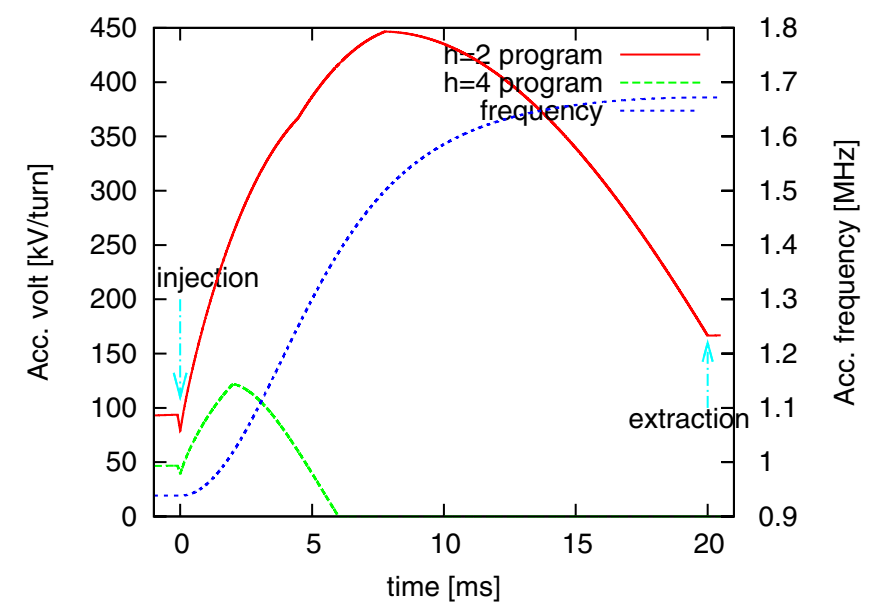

FIG. 15. (Color) Dual-harmonic acceleration pattern (voltage and frequency). The maximum ratio of $(h=4)$ is 0.5 .

reason to fit the beam conditions. As described in Sec. I, the voltage ratio of the accelerating $\mathrm{rf}(h=2)$ and the second harmonic $(h=4)$ varies rapidly in the cycle. From the beam injection to $2 \mathrm{~ms}$ after injection, the ratio is about 0.5 . After $2 \mathrm{~ms}$ the ratio becomes smaller and finally at $6 \mathrm{~ms}$ the voltage of $(h=4)$ goes to zero. The phase of the second harmonic is programmed to follow the synchronous phase of the beam by referring to the fundamental rf voltages.

The comparisons between the programmed and the generated voltages are shown in Fig. 16. The measured voltage is the vector sum of the ten cavities. The generated voltages of the $(h=2)$ and $(h=4)$ follow the programs nicely. One can notice that a small amount of the second harmonic voltage remains after $6 \mathrm{~ms}$, while the program voltage is zero. This is mainly because of the distortions by the transistor driver amplifier. Also, the distortion of the tubes and the unbalance of the tubes of the push-pull amplifiers can generate the unwanted second harmonic voltage. The

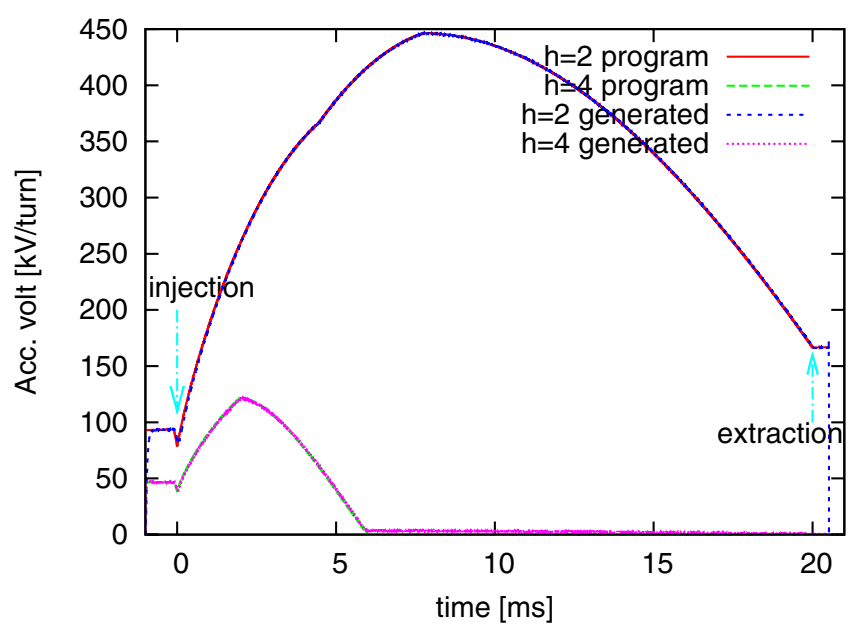

FIG. 16. (Color) Program and generated voltages. 


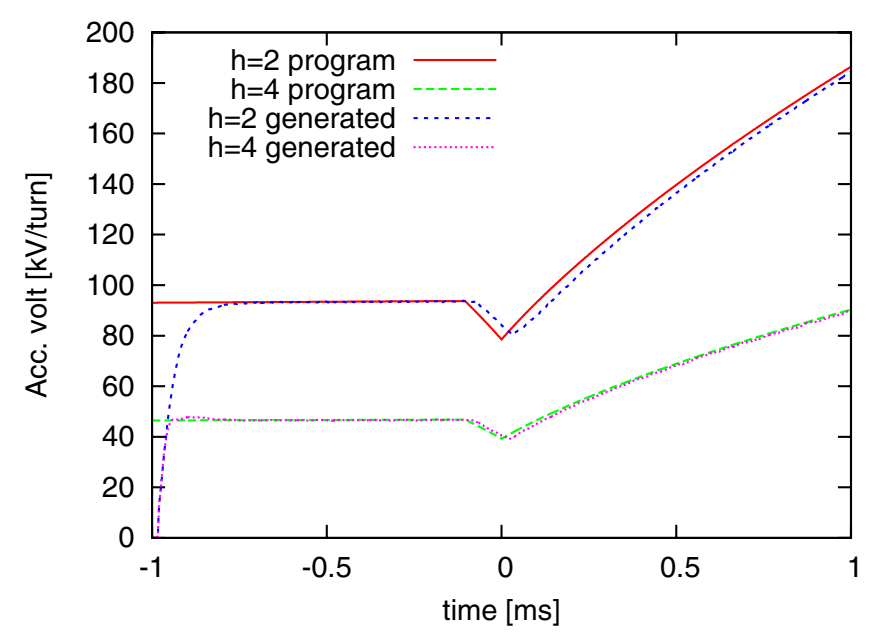

FIG. 17. (Color) Program and generated voltages, near the injection period.

amplitude of the remaining $(h=4)$ component is a few percents and the level of distortion gives very small effects on the beam acceleration.

The magnified plot near the injection is shown in Fig. 17. In this period, the error is relatively large, 6 and $2 \mathrm{kV}$ maximum for $(h=2)$ and $(h=4)$, respectively. In this period, these levels of errors are tolerable.

\section{Beam acceleration with the dual-harmonic rf}

The beam commissioning of the RCS was started on September 2007. As of March 2008 the acceleration of $10^{13}$ protons up to $3 \mathrm{GeV}$ is achieved with a low repetition rate. We have performed a preliminary test of accelerating the proton beam with applying the second harmonic rf voltage. The voltage patterns in Fig. 15 are used for $(h=$ 2) and $(h=4)$.

The test beam conditions are as follows: (i) the macropulse width of linac beam is $120 \mu \mathrm{s}$; (ii) the chopping

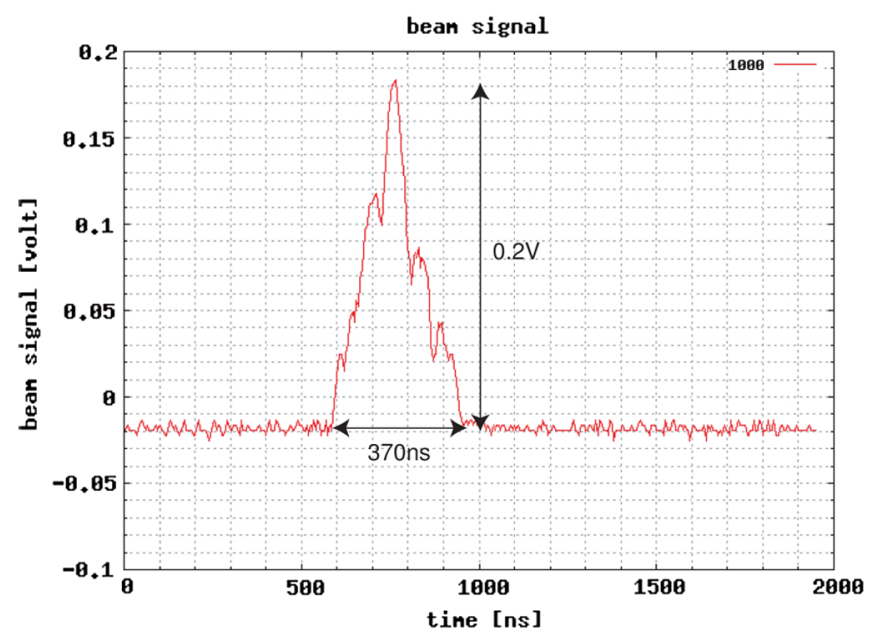

FIG. 18. (Color) Bunch shape without the second harmonic rf. The full width of the bunch is $370 \mathrm{~ns}$.

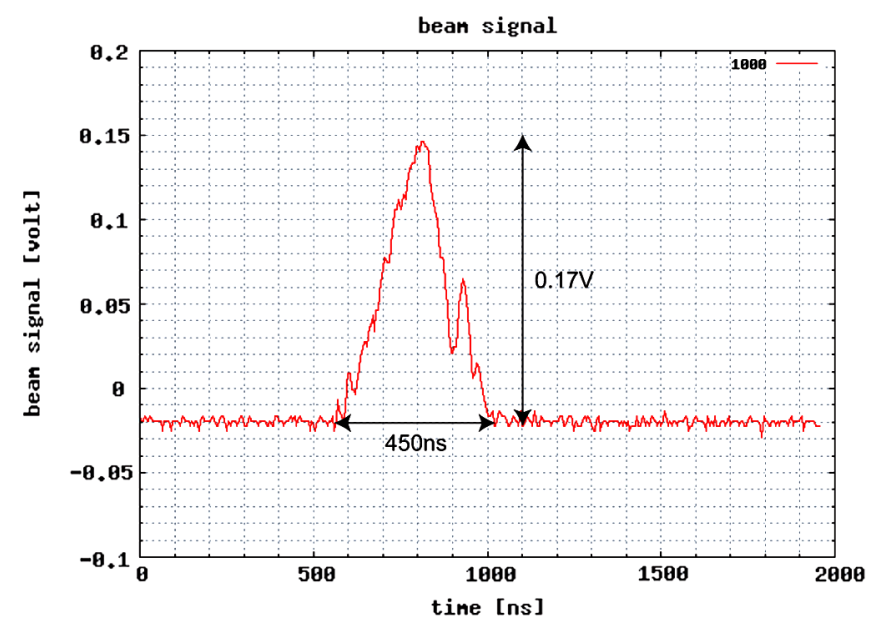

FIG. 19. (Color) Bunch shape with the second harmonic rf and $0.1 \%$ of momentum-offset injection. The full width of the bunch is $450 \mathrm{~ns}$.

width is $560 \mathrm{~ns}$; (iii) the peak current of the linac beam is $25 \mathrm{~mA}$; (iv) one bucket of the RCS rf buckets is filled (onebunch operation); (v) the number of protons in the bunch is about $4 \times 10^{12}$. We compared the bunch shape signal picked up by the wall current monitor (WCM) in two cases, without and with the second harmonic rf voltages. In the case of applying the second harmonic, we also employed the momentum-offset injection scheme $(+0.1 \%$ offset.)

In Figs. 18 and 19, the bunch shapes at turn 1000 (2 ms after the injection) with and without the second harmonic rf are shown, respectively.

The full width of the bunch is $450 \mathrm{~ns}$ with the second harmonic rf while the width is 370 ns without the second harmonic. Reduction of the peak beam current by about $15 \%$ is observed. The test is still preliminary, however, clearly the bunch is lengthened by applying the second harmonic $\mathrm{rf}$ and the bunching factor of the beam is improved.

The macropulse width of $120 \mu \mathrm{s}$ is still much shorter than the design $(500 \mu \mathrm{s})$. Because the synchrotron frequency at the injection is about $3.5 \mathrm{kHz}$, the longitudinal painting was not sufficient. Thus, the bunch contains filaments so that many small peaks appear in the beam signal from WCM. For the detailed studies of how the second harmonic rf performs, we need the full design width of the macropulse.

\section{CONCLUSION AND FUTURE WORK}

We summarize the article as follows: (i) Dual-harmonic rf is useful to accelerate the ultrahigh intensity proton beam in the J-PARC RCS. To realize the dual-harmonic operation, the dual-harmonic voltage control system (dualharmonic AVC) is necessary. (ii) A full-digital LLRF control system for J-PARC RCS, including dual-harmonic $\mathrm{AVC}$ has been designed and built. (iii) The dual-harmonic 
AVC picks up the amplitude of each harmonic, $(h=2)$ and $(h=4)$, and regulates the amplitude using PID control. (iv) Various tests have been performed. The voltage ratio is controlled as expected. The generated voltages follow the programs with tolerable errors. (v) The beam commissioning of the J-PARC RCS has been started. A preliminary beam test with the dual-harmonic has been performed. The bunching factor is improved by applying the second harmonic. Further studies are to be done with a wider macropulse of the linac.

The simulation shows that the higher ratio of the second harmonic, $80 \%$ at maximum, is the optimum for the beam with full designed intensity. With such a high ratio, the rf bucket shape is no longer flat but has a constriction, which introduces two stable points in the bucket. In this case, the tolerance of the phase error of the second harmonic becomes smaller to avoid an unbalance of the bunch distribution. We are interested in the response and the stability of the dual-harmonic AVC under the heavy beam loading, which causes a phase error of the cavity voltages on both the fundamental accelerating rf and the second harmonic.

\section{ACKNOWLEDGMENTS}

We would like to thank C. Ohmori, M. Yamamoto, M. Nomura, M. Toda, E. Ezura, S. Anami, K. Hara, and K. Katsushi for fruitful discussions and comments. We are grateful for the excellent construction work of the LLRF hardware by the company, Nichizou Denshi Seigyo (NDS).
We would like to especially thank two engineers of NDS, Y. Yamamoto and N. Kawamura. The beamcommissioning group headed by $\mathrm{H}$. Hotchi provided the stable beam condition which was necessary for carrying out the beam test of the dual-harmonic AVC, and we would like to thank the J-PARC RCS members.

[1] JHF accelerator design study report, KEK Report No. 9716, 1997.

[2] Accelerator technical design report for J-PARC, JAERITECH 2003-044, 2003.

[3] M. Yamamoto et al., in Proceedings of the 8th European Particle Accelerator Conference, Paris, 2002 (EPS-IGA and CERN, Geneva, 2002), pp. 1073-1075.

[4] A. Schnase et al., in Proceedings of the 10th European Particle Accelerator Conference, Edinburgh, Scotland, 2006 (EPS-AG, Edinburgh, Scotland, 2006), pp. 14651467.

[5] F. Tamura et al., in Proceedings of the 10th European Particle Accelerator Conference, Edinburgh, Scotland, 2006 (EPS-AG, Edinburgh, Scotland, 2006), pp. 13191321.

[6] A. Schnase et al., Phys. Rev. ST Accel. Beams 8, 122001 (2005).

[7] M. Yoshii et al., in Proceedings of the 2007 Particle Accelerator Conference, Albuquerque, New Mexico, 2007 (IEEE, Albuquerque, New Mexico, 2007), pp. 1511-1513. 